\title{
In vitro metabolic capacity of carbohydrate degradation by intestinal microbiota of adults and pre-frail elderly
}

Ran $A n \mathbb{D}^{1 凶}{ }^{凶}$, Ellen Wilms ${ }^{2}$, Madelon J. Logtenberg ${ }^{3}$, Mara P. H. van Trijp ${ }^{4}$, Henk A. Schols $\mathbb{D}^{3}$, Ad A. M. Masclee ${ }^{2}$, Hauke Smidt ${ }^{1}$, Daisy M. A. E. Jonkers ${ }^{2}$ and Erwin G. Zoetendal (iD ${ }^{1}$

(c) The Author(s) 2021

Globally increased life expectancy strongly triggered interest to delay the onset of frailty, which has been associated with alterations in compositional and functional characteristics of intestinal microbiota. In the current study, we used an in vitro batch incubation model to compare the metabolic capacity of the faecal microbiota of adults $(n=6)$ versus pre-frail elderly $(n=6)$ to degrade various glycosidic carbohydrates, including galacto-oligosaccharides, 2'-fucosyllactose, chicory fructo-oligosaccharides and inulin, and isomalto/malto-polysaccharides. The in vitro metabolic capacity was also compared with an in vivo GOS intervention study based on the same subjects. Analysis of $16 \mathrm{~S}$ rRNA gene sequences and metabolites revealed distinct portions of variation in overall microbiota and metabolite composition during incubation being explained by individuality of the subjects and carbon source. In addition, the age group of the subjects also had significant impact on microbiota variation, carbohydrate degradation and metabolite production. This was accompanied by elevated increase in the relative abundance of Bifidobacterium in the microbiota of adults compared to that of pre-frail elderly and significantly decreased effectiveness to degrade galactooligosaccharides by the latter group. Altogether, the carbohydrate degradation in elderly was different compared to adults, with some carbohydrates showing decreased degradation rates. Longer interventions periods may be required to enhance bifidobacterial abundance in the microbiota of pre-frail elderly and thereby to obtain associated prebiotic health benefits.

ISME Communications; https://doi.org/10.1038/s43705-021-00065-5

\section{INTRODUCTION}

The human intestinal tract is inhabited by a large number of microorganisms, collectively called the intestinal microbiota. The intestinal microbiota plays an important role in human health as it converts indigestible carbohydrates into metabolites, which are largely absorbed by the intestine, but it also produces essential vitamins and affects immune system development and functioning [1].

Ageing is associated with alterations in intestinal physiology and functionality, such as increased oro-caecal and colonic transit time, thereby affecting the inhabiting microorganisms [2]. Many studies have investigated the changes in faecal microbial composition during ageing by comparing the microbiota of adults and elderly [3-6]. Notably, studies investigating the intestinal microbial composition of centenarians demonstrated that parameters related to health status rather than chronological ageing per se were associated to the changes in microbiota composition during ageing [4, 7]. Further, it should be noted that age cut-off for elderly, varying from $60-80$ years, is inconsistent between studies making comparative analyses between studies difficult [8]. As such, it is not surprising to have contradictory findings being reported in terms of changes in microbiota composition during ageing [9]. Nevertheless, compared to adults, the intestinal microbiota of elderly has frequently been reported to contain higher levels of streptococci and Enterobacteriaceae as well as lower levels of Bifidobacterium, with differences being more pronounced in frail or comorbid elderly [10]. In addition, comparative microbiota composition analysis revealed a lower level of Bifidobacterium in pre-frail elderly compared to healthy adults [11]. Rampelli et al. employed a metagenomic approach and revealed that the microbiota of elderly had decreased saccharolytic potential, as shown by a loss of genes involved in carbohydrate metabolism and decreased number of genes coding for enzymes involved in short chain fatty acid production, in comparison to the microbiota of young adults [12]. Nevertheless, as it has been shown that both within and across microbial groups, microorganisms demonstrate high levels of functional redundancy [13], the impact of these reduced gene numbers on intestinal function has yet to be demonstrated.

While DNA-based approaches to study the microbiota can be used to predict its functional potential, the actual activity of microorganisms depends on different biotic and abiotic conditions [14]. Moreover, microbes are very versatile and can quickly adapt to changes in their living environment [15]. Hence, DNAbased approaches do not provide information about actual metabolic activity of the microbiota towards the degradation of

\footnotetext{
${ }^{1}$ Laboratory of Microbiology, Wageningen University \& Research, Wageningen, The Netherlands. ${ }^{2}$ Division Gastroenterology-Hepatology, Department of Internal Medicine, NUTRIM School of Nutrition and Translational Research in Metabolism, Maastricht University Medical Centre+, Maastricht, The Netherlands. ${ }^{3}$ Laboratory of Food Chemistry, Wageningen University \& Research, Wageningen, The Netherlands. ${ }^{4}$ Nutrition, Metabolism \& Genomics Group, Division of Human Nutrition and Health, Wageningen University \& Research, Wageningen, The Netherlands. ${ }^{凶}$ email: ran.an@wur.nl
}

Received: 28 December 2020 Revised: 27 September 2021 Accepted: 7 October 2021

Published online: 28 October 2021 
Table 1. Characteristics of adults $(n=6)$ and elderly $(n=6)$ included in this study.

\begin{tabular}{|llll|}
\hline & $\begin{array}{l}\text { Adults } \\
(\boldsymbol{n}=\mathbf{6})\end{array}$ & $\begin{array}{l}\text { Elderly } \\
(\boldsymbol{n}=\mathbf{6})\end{array}$ & $\boldsymbol{P}$ value \\
\hline Age (yrs, mean \pm SD) & $34.0 \pm 4.2$ & $72.8 \pm 3.0$ & $<0.001$ \\
\hline Sex $(\%$ female) & 50 & 50 & 1 \\
\hline BMI $\left(\mathrm{kg} / \mathrm{m}^{2}\right.$, mean \pm SD) & $24.2 \pm 3.7$ & $26.5 \pm 3.0$ & 0.277 \\
\hline Alcohol (\%) & 83.3 & 83.3 & 1 \\
\hline Smoking (\%) & 0 & 16.7 & 1 \\
\hline Anticoagulation use (\%) & 0 & 16.7 & 1 \\
\hline Antispasmodics (\%) & 0 & 16.7 & 1 \\
\hline $\begin{array}{l}\text { Habitual dietary fibre intake } \\
\text { (g/day) }\end{array}$ & $22.4 \pm 6.5$ & $26.6 \pm 2.5$ & 0.208 \\
\hline
\end{tabular}

Between groups, parameters, i.e., age, BMI, were tested with independent Student's $t$ test. Percentage of sex, alcohol consumption, smoking, medication use and dietary fibre intake were compared using Fisher's exact test between groups.

$B M I$ body mass index, SD standard deviation, yrs years of age.

specific carbohydrates. Therefore, in vitro incubations under conditions simulating the intestinal tract can be used to verify functional prediction and to provide refined information regarding to functional capacity.

Given the differences in microbiota composition between elderly and younger adults, notably with respect to the number of bifidobacteria, this study aimed to compare the metabolic capacity of microbiota in adults and elderly in response to carbohydrates of different molecular structure, including galactooligosaccharides (GOS) [16], 2'-fucosyllactose (2'-FL) [17, 18], chicory fructo-oligosaccharides (FOS; synonym oligofructose), chicory inulin [19] and isomalto/malto-polysaccharides (IMMP) [20], which are all known to be utilized by the intestinal microbiota and often considered as bifidogenic. We hypothesized that the metabolic capacity of pre-frail elderly microbiota is lower compared to that of adults, in terms of carbohydrate degradation and metabolite production.

\section{MATERIALS AND METHODS \\ Study setup}

Six adults and six elderly, who were included in a previously conducted in vivo GOS intervention study [11], donated their faecal material for the current study (Fig. S1) at their first visit or at least 4 weeks after the intervention period. Each participant defecated into a stool collector (Excretas Medical BV, Enschede, the Netherlands). Directly after defecation, faecal material was divided into two portions. A small portion ( $0.5 \mathrm{~g})$ was frozen immediately. The remaining faeces was anoxically cryo-conserved and used as inoculum for the in vitro incubations. The viability of different microbial groups in the anoxically cryo-conserved faecal material was determined with propidium monoazide (PMA) dye. The in vitro incubations lasted for $24 \mathrm{~h}$ with samples collected in duplicate to compare microbiota composition, carbohydrate degradation and metabolite production between age groups (adults vs elderly). The degrading capacity for two typical bifidogenic carbohydrates, i.e., GOS and 2'-FL, was determined for the microbiota of all six adults and six elderly and compared to a noncarbohydrate control. To further extend these experiments, we also studied the degradation of other typical bifidogenic carbohydrates, i.e. FOS, inulin, and IMMP, using the faecal inocula of three adults and three elderly for which sufficient material was still available.

\section{Participants}

The six adults (20-30yrs) and six elderly participants (70-85 yrs) of the intervention study [11] were randomly contacted and participated in the current study, who differed significantly in age, but not in sex, BMI, alcohol consumption, smoking, medication use or dietary fibre intake (Table 1). None of the participants took acid inhibitors (e.g., proton pump inhibitors), nor antibiotics 90 days prior to the study, nor did any of the participants have a chronic disorder or major surgery, as these factors potentially could have limited participation, completion of the study, or interfered with the study outcomes. Detailed description of the inclusion and exclusion criteria has been provided previously [11]. Subject codes as shown in the results were randomly assigned in the data analysis phase and cannot be traced back to individual subjects without the specific randomization key. The study was approved by the medical Ethics Committee of the Maastricht University Medical Center+ and registered in the US National Library of Medicine (http://www.clinicaltrials.gov) with the registration number NCT03077529 [11].

\section{Dietary intake}

Participants in the current study completed the dietary records on 3 consecutive days, after instructed to record their food, beverage and dietary supplement intake based on standard household units. Their nutrient intake was analyzed using the online dietary assessment tool of The Netherlands Nutrition Centre (www.voedingcentrum.nl).

\section{Carbohydrates}

Five different carbohydrates, i.e., GOS, 2'-FL, FOS, inulin and IMMP were used as sole carbon sources in this study. GOS and the human milk oligosaccharide 2'-FL (Fuca1-2Galß1-4GIc) were kindly provided by Friesland Campina (Amersfoort, The Netherlands). In order to mimic the actual portion of GOS utilized by intestinal microbiota, purified GOS with $<3 \%$ monomers and lactose was used. Size distribution of mono- and oligomers was as follows: $2.4 \%$ degree of polymerization (DP) 1, $11.3 \%$ DP2, $41.8 \%$ DP3, 25.6\% DP4, 12.1\% DP5, 4.6\% DP6, 1.4\% DP7, 0.34\% DP8, 0.11\% DP9. FOS and inulin were kindly provided by Sensus (Roosendaal, the Netherlands). FOS or oligofructose (Frutalose OFP) is derived from partial enzymatic hydrolysis of inulin from chicory and consisted for $92 \pm 2 \%$ of FOS (DP2-10) and for $8 \pm 2 \%$ of a mixture of fructose, glucose and sucrose. Long-chain inulin (Frutafit TEX!), termed as inulin in the current study, is also derived from chicory, comprising $\geq 99.5 \%$ inulin (DP2 to 60 , average chain length $\geq 22$ monomers), and $\leq 0.5 \%$ mixture of fructose, glucose and sucrose. The IMMP is IMMP-92 (AVEBE, Groningen, the Netherlands) which is a novel indigestible a-glycan derived from starch, with $92 \%$ of $a-(1 \rightarrow 6)$ glycosidic linkages [21].

\section{Faecal sample collection and storage}

To store and transport freshly defecated faeces under anoxic conditions, Anaerocult ${ }^{\circ} \mathrm{A}$ mini (Merck KGaA, Darmstadt, Germany) was activated with $10 \mathrm{ml}$ nuclease-free water (Promega, Madison, WI, US), and placed next to the faeces in the stool collector before the lid was closed to create an anoxic atmosphere. Afterwards, the stool collector and two open bags of AnaeroGen (AnaeroGen ${ }^{\mathrm{TM}} 3.5 \mathrm{~L}$ Sachet, Thermo Scientific, Waltham, Massachusetts, US) were put into an anoxic box (AnaeroPack ${ }^{\mathrm{TM}} 7.0 \mathrm{~L}$ Rectangular Jar, Thermo Scientific) and stored at $4{ }^{\circ} \mathrm{C}$ until transportation. Samples were transported on ice from Maastricht to Wageningen University \& Research within $9 \mathrm{~h}$. After arrival, the anoxic box was transported immediately into the anaerobic chamber (MK3 Workstation, Don Whitley, UK), filled with an atmosphere of $4 \% \mathrm{H}_{2}$ and $96 \% \mathrm{~N}_{2}$. For each donated sample, every $17.5 \mathrm{~g}$ faeces were mixed with $7.5 \mathrm{~g}$ dialysate (Tritium Microbiologie, Eindhoven, the Netherlands), $35.7 \mathrm{~g}$ nuclease-free water and $9.8 \mathrm{ml}$ glycerol. The mixed faecal slurry was transferred into a serum bottle and sealed with a butyl rubber stopper and metal crimp cap inside the anaerobic chamber, and afterwards stored at $-80^{\circ} \mathrm{C}$.

\section{In vitro incubations}

Anoxically cryo-conserved faecal inoculum was defrosted and transferred to an anaerobic chamber filled with an atmosphere of $96 \% \mathrm{~N}_{2}$ and $4 \% \mathrm{H}_{2}$ (BACTRON 300, Shel Lab, Cornelius, Oregon, US). Using standard ileal efflux medium (Tritium Microbiologie) [20], incubation was done with one of the carbohydrates $(10 \mathrm{mg} / \mathrm{ml})$ and $10 \%(\mathrm{v} / \mathrm{v})$ faecal inoculum in duplicate, while incubations without faecal inoculum or without carbohydrates, respectively, served as controls. Specifically, every $1 \mathrm{~L}$ of medium comprised $400 \mathrm{ml} \mathrm{BCO}(60 \mathrm{~g} / \mathrm{L}$ casein, $60 \mathrm{~g} / \mathrm{L}$ bacto peptone and $1 \mathrm{~g} / \mathrm{L}$ ox bile), $16 \mathrm{ml}$ salts solution $(156.3 \mathrm{~g} / \mathrm{L}$ di-potassium hydrogen phosphate, $281.3 \mathrm{~g} / \mathrm{L}$ sodium chloride, $28.13 \mathrm{~g} / \mathrm{L}$ calcium chloride dihydrate, $0.31 \mathrm{~g} / \mathrm{L}$ iron (II) sulfate heptahydrate, $0.63 \mathrm{~g} / \mathrm{L}$ hemin porcine), $4 \mathrm{ml}$ cystein. $\mathrm{HCl}$ solution, $0.8 \mathrm{ml}$ vitamin $\operatorname{mix}(1 \mathrm{mg} / \mathrm{L}$ menadion, $2 \mathrm{mg} / \mathrm{L} \mathrm{D}(+)$ biotine, 0.5 $\mathrm{mg} / \mathrm{L}$ Vitamin $\mathrm{B} 12,10 \mathrm{mg} / \mathrm{L} \mathrm{D}(+)$ pantothenate, $5 \mathrm{mg} / \mathrm{L}$ aminobenzoic acid, $4 \mathrm{mg} / \mathrm{L}$ thiamine $\mathrm{HCL}$ and $5 \mathrm{mg} / \mathrm{L}$ nicotinamide adenine dinucleotide free 
acid) and $100 \mathrm{ml}$ MES (1 M pH 6.0). Except for the to-be-studied carbohydrates (i.e., IMMP, short chain \& long chain inulin, 2'-FL and GOS), no additional carbohydrates were added in the fermentation medium. Ten $\mathrm{ml}$ batch incubation bottles were used in the current study, and filled with $6 \mathrm{ml}$ fermentation medium. Cultures were incubated at $37^{\circ} \mathrm{C}$ on a rotary shaker at $200 \mathrm{rpm}$ for $24 \mathrm{~h}$.

\section{Sample collection}

Samples were collected 0, 4, 10 and $24 \mathrm{~h}$ after inoculation (Fig. S2). Specifically, at each time point, two incubation bottles (the duplicate) per treatment were sacrificed for sample collection. The headspace gas was sampled first to determine $\mathrm{H}_{2}$ and $\mathrm{CH}_{4}$ production. Three aliquots of $1 \mathrm{ml}$ culture were then distributed into $1.5 \mathrm{ml}$ Eppendorf tubes. One of these aliquots was heated at $100^{\circ} \mathrm{C}$ for $5 \mathrm{~min}$ to determine carbohydrates in the supernatant. Afterwards, all aliquots were centrifuged at $4{ }^{\circ} \mathrm{C}$ at $18,600 \mathrm{rcf}$ for $10 \mathrm{~min}$. The supernatants from the other two unheated tubes were stored at $-20^{\circ} \mathrm{C}$ for metabolite measurement, while the remaining pellets were stored at $-80^{\circ} \mathrm{C}$ for microbiota analysis.

\section{Carbohydrate, gas, and metabolite measurements}

Degradation of GOS, 2'-FL, FOS, inulin and IMMP was determined using High-Performance Anion Exchange Chromatography (HPAEC) with Pulsed Amperometric Detection (PAD). Specifically, samples taken during the incubation were diluted and centrifuged for $15 \mathrm{~min}$ at $18,600 \mathrm{rcf}$. Ten microlitres of supernatant was injected to an ISC5000 HPLC system (Dionex, Sunnyvale, CA, US), which was composed of a CarboPac PA-1 column ( $250 \mathrm{~mm} \times 2 \mathrm{~mm}$ ID), a CarboPac PA guard column $(25 \mathrm{~mm} \times 2 \mathrm{~mm}$ ID) and an ISC5000 ED detector (Dionex) in the PAD mode. Detailed description of gradients and dilution factors are provided in the supplementary information. The degradation and size of the large IMMP molecules was also determined using High Performance Size Exclusion Chromatography as described previously [20].

Headspace gas composition was measured using a CompactGC gas chromatograph (Global Analyser Solutions, Breda, The Netherlands), equipped with a Carboxen PDD precolumn (pressure: $200 \mathrm{kPa}$, split flow: $20 \mathrm{ml} / \mathrm{min}$, column oven: $90^{\circ} \mathrm{C}$, valve oven: $80^{\circ} \mathrm{C}$ ) with a carrier gas flow of $20 \mathrm{ml} / \mathrm{min}$ and a TCD column (pressure: $200 \mathrm{kPa}$, split flow: $10 \mathrm{ml} / \mathrm{min}$, column oven: $80^{\circ} \mathrm{C}$, valve oven: $80^{\circ} \mathrm{C}$ ).

Concentration of organic acids was determined by High Performance Liquid Chromatography (HPLC), using a SUGAR SH1821 column (SHODEX, Tokyo, Japan). The column was operated at $54{ }^{\circ} \mathrm{C}$ with a flow rate of $0.8 \mathrm{ml} /$ min, using $0.01 \mathrm{~N} \mathrm{H}_{2} \mathrm{SO}_{4}$ as eluent. The compounds were detected by an RID-20A (Shimadzu, Kyoto, Japan) refractive index detector at a temperature of $40^{\circ} \mathrm{C}$. Four hundred $\mu \mathrm{l}$ of collected supernatant was mixed with $600 \mu \mathrm{l}$ of $10 \mathrm{mM}$ DMSO in $0.01 \mathrm{~N} \mathrm{H}_{2} \mathrm{SO}_{4}$, and $10 \mu \mathrm{l}$ of this mixture was injected for analysis. All analytical measurement data were processed using Chromeleon ${ }^{\mathrm{TM}}$ Chromatography Data System (CDS) Software (Thermo Scientific).

\section{Microbiota composition analysis}

The microbiota composition in faecal- and batch incubation samples was determined by sequencing of barcoded $16 \mathrm{~S}$ ribosomal RNA (rRNA) gene amplicons (details in Supplementary information). In short, total DNA was obtained from the collected pellet by repeated bead beating and purification with a Maxwell 16 Instrument (Promega, Leiden, The Netherlands). The V4 region of the 16S rRNA gene was amplified in triplicate using barcoded 515F [22] - 806R [23] primers and total bacterial DNA as template as described previously [24]. An equimolar mix of purified PCR products was sent for Illumina Hiseq2500 ( $2 \times 150 \mathrm{bp}$ ) sequencing (Eurofins Genomics, Konstanz, Germany). Raw sequence data were processed using NG-Tax 1.0 with default settings [25]. Taxonomy was assigned based on SILVA database version $128[26,27]$. A detailed description is provided in the supplementary information. The raw sequence data has been uploaded to the European Nucleotide Archive with accession number PEJEB41341. In addition, the total genomic DNA was used for total bacterial quantification as it has been described earlier [11].

\section{Viability measurements}

The fraction of viable microbes in the anoxically cryo-conserved faeces was determined with PMA dye, a photoreactive dsDNA-binding dye that only penetrates the envelop of dead cells [28]. Briefly, $1 \mathrm{ml}$ of anoxically cryoconserved faecal inoculum from each donor was mixed with $2.5 \mu \mathrm{l}$ of 20 mM PMA dye (Biotium, Inc., Fremont, CA, USA), and incubated at room temperature for $5 \mathrm{~min}$ in the dark, followed by treatment with a PMA-Lite ${ }^{T M}$ LED photolysis device (Biotium, Inc.) for 15 min. Subsequently, samples were centrifuged at $4{ }^{\circ} \mathrm{C}$ for $10 \mathrm{~min}$ ( $1500 \mathrm{rcf}$ ). The pellet was used for microbiota composition analysis.

\section{Statistical analysis}

All statistical analyses were conducted in $\mathrm{R}$ (R-3.6.3). Relative abundance of microbial taxa was calculated based on $16 \mathrm{~S}$ rRNA gene sequence read counts. The microbial diversity (Inverse Simpson) and richness (Phylogenetic Diversity) were calculated based on amplicon sequence variants, which were also used to calculate distance matrices. Permutational multivariate analysis of variance (PERMANOVA) was performed based on weighted and unweighted UniFrac distance matrices. Principal coordinate analysis was used to visualize the microbiota composition variation between samples [29]. Microbiota variation partitioning was assessed by fitting environmental variables (i.e., age group, sampling time point and type of carbohydrate) to weighted and unweighted UniFrac distance matrices, using the adonis function in the vegan package [30]. To compare and contrast alterations in microbiota composition with different carbohydrates versus non-carbohydrate control during the incubation, we used principal response curve analysis to identify genera which fit best (weights $>0.05$ ) to explain the observed difference, using the prc function in the vegan package [30]. As for the metabolite data, redundancy analysis (RDA) in combination with Monte Carlo permutation was performed to assess to what extent explanatory variables, i.e., incubation time, subject- and carbohydrate-specificity, could explain the overall variation in metabolite data, using the rda function in the vegan package [30]. To assess the effect of age group (adult vs elderly) on the degradation of carbohydrates/concentration of metabolites during incubation, we analyzed the data using two-way mixed ANOVA, with one between-subjects factor (age group) and one within-subjects factor (incubation time), using the anova_test function in the rstatix package [31]. False discovery rate (FDR) correction according to the Benjamini-Hochberg procedure was applied for multiple testing when applicable. A corrected $P$ value $<0.05$ was considered to indicate significant difference.

\section{RESULTS}

\section{Metabolite production from carbohydrate incubation}

In vitro batch incubations were used to assess the metabolic capacity of the faecal microbiota of adults and elderly towards different carbohydrates. RDA revealed significant contribution of age group (2.16\%), incubation time (34.66\%), subject identity $(16.31 \%)$ and type of carbohydrate $(8.65 \%)$ to the overall variation in metabolite data (Fig. 1A), in line with what was observed for microbiota composition (Fig. 1B, C). RDA performed separately for the different time points showed that age group (3.17-6.63\%) contributed to the variation in metabolite data throughout the incubation period (Fig. 1D). Over time the relative contribution of subject identity to explaining metabolite variation decreased, while contribution of carbohydrates increased (Fig. 1D), which is in line with changes in microbiota composition (Fig. 1E, F). Nevertheless, from 10 to $24 \mathrm{~h}$, variation explained by carbohydrate decreased (Fig. 1D), which may be attributed to the depletion of some carbohydrates such as 2'-FL and FOS after $10 \mathrm{~h}$ (Fig. 2). Although buffered with MES, we observed a slight decrease of $\mathrm{pH}$ over time from approximately $6.4-5$ as a result of carbohydrate fermentation, with slightly higher rates of acidification for FOS and IMMP for some subjects (Fig. S3). The incubation without additional carbohydrates added (i.e., the control in Fig. S3) showed a slight increase of $\mathrm{pH}$ over time due to the utilization of protein and subsequent production of ammonia.

Zooming in on the effect of age group on the concentration of the different metabolites (Table 2), for the GOS incubation, the concentrations of propionate and butyrate differed significantly $(P<0.001$ and $P=0.048$, respectively) between age groups, with significantly higher concentrations of propionate and butyrate in elderly compared to those in adults $24 \mathrm{~h}$ after inoculation. In contrast, the concentration of acetate did not differ significantly between adults and elderly. 

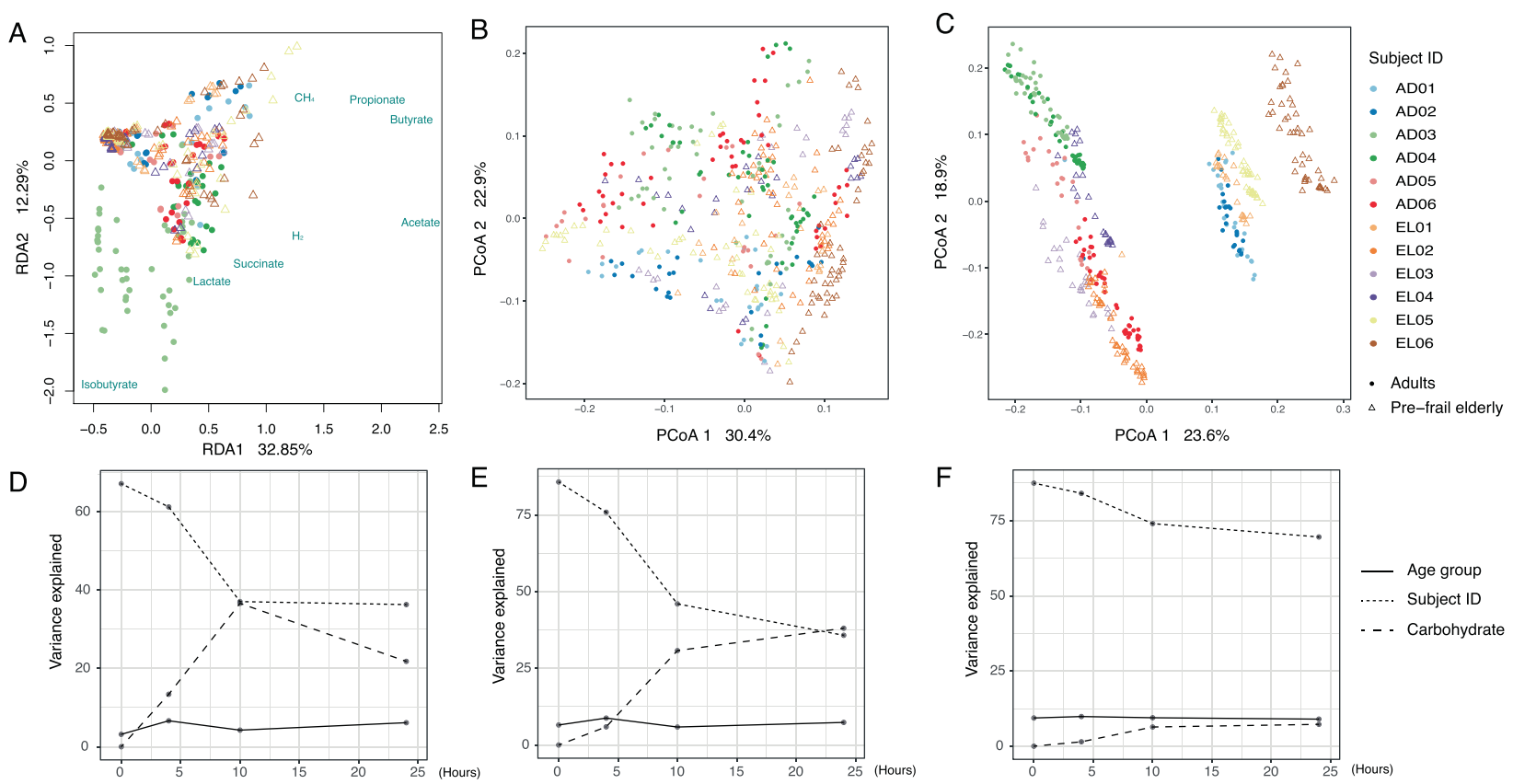

Fig. 1 Microbiota and metabolite variation in the dataset. A RDA based on total metabolite data. PCoA based on B weighted UniFrac and C unweighted UniFrac distance matrices of all incubation samples. D variation in metabolites that can be explained by age group, subjects or carbohydrates at $0,4,10$ and $24 \mathrm{~h}(\mathbf{E}, \mathbf{F})$ Variation in microbiota composition that can be explained by age group, subjects or carbohydrates, based on (E) weighted and (F) unweighted UniFrac distance matrices at $0,4,10$ and $24 \mathrm{~h}$. Both duplicate samples were included for the analysis and demonstration. AD adult, EL elderly, PCoA principal coordinate analysis, RDA redundancy analysis.

In response to $2^{\prime}-\mathrm{FL}$, the concentration of butyrate differed significantly between age groups $(P=0.048)$, with significantly higher concentration of butyrate at $24 \mathrm{~h}$ in elderly, compared to that in adults (Table 2). Besides, the concentration of acetate was significantly lower in elderly at $4 \mathrm{~h}(P=0.049)$ and $10 \mathrm{~h}(P=0.003)$ compared to that in adults.

As for FOS, the concentrations of propionate and butyrate were significantly higher in elderly after $24 \mathrm{~h}$ of incubation compared to those in adults (Table 3). Concurrently, the concentration of acetate did not differ between elderly and adults.

For incubations with inulin and IMMP, the concentration of $\mathrm{CH}_{4}$ was significantly higher in elderly at 4,10 and $24 \mathrm{~h}$ compared to that of adults. In addition, the concentration of butyrate at $24 \mathrm{~h}$ was significantly higher in elderly compared to that in adults when IMMP was used.

For none of the carbohydrates, incubations with microbiota of adults and elderly differed significantly in the concentration of lactate, succinate or isobutyrate (Tables 2 and 3). However, compared to the other carbohydrates, the concentration of succinate was higher in response to IMMP (Tables 2 and 3), which coincided with predominance of Bacteroides in the microbiota (Figs. S4E, S5E, S6B and S7), indicating succinate as the main product of IMMP utilization by Bacteroides. Furthermore, the faecal microbiota of only two out of six adults and three out of six elderly demonstrated production of $\mathrm{CH}_{4}$, in line with detection of methanogens, i.e., Methanobrevibacter (Fig. S8).

\section{Degradation kinetics of GOS, FOS, 2'-FL, inulin and IMMP during incubation}

Considering all DPs of GOS as a whole, the microbiota of elderly was significantly slower in GOS degradation compared to the microbiota of adults ( $P=0.041$, Fig. 2A, DP distribution in Fig. S9). Zooming in on specific DPs, the microbiota of elderly was especially slower in the degradation of DP2 $(P=0.047)$ and DP3 $(P=0.068)$, compared to the microbiota of adults (Fig. $2 \mathrm{~A})$.

As for 2'-FL, the microbiota of adults and elderly did not differ significantly in its degradation (Fig. 2B), although subjects with lower abundance/no faecal Bifidobacterium at the start and during the incubation (i.e., EL02 and EL06, Fig. S6A) were remarkably slower in 2'-FL degradation, as compared to subjects with higher relative abundance of Bifidobacterium, like AD03 and AD05 (Fig. S6A).

FOS is composed of fructose (F) oligosaccharides with or without a terminal glucose $(G)$ residue. Specifically, in the current study, FOS contained 2.1\% F, 1.8\% $\mathrm{F}_{2}, 3.4 \% \mathrm{GF}, 2.8 \% \mathrm{GF}_{2}, 6.0 \% \mathrm{GF}_{3}$, $27.5 \% \mathrm{~F}_{3}, 10.0 \% \mathrm{GF}_{4}, 21.4 \% \mathrm{~F}_{4}, 7.6 \% \mathrm{GF}_{5}, 8.9 \% \mathrm{~F}_{5}, 2.9 \% \mathrm{GF}_{6}$ (Fig. S10). In contrast to GOS, the microbiota of adults and elderly did not differ significantly in FOS degradation when considering all oligosaccharides as a whole $(P>0.05)$, nor after zooming in on specific oligosaccharides (Fig. 2C). In addition, degradation of FOS coincided with formation of fructose mono- (F) and dimer (F2) after $4 \mathrm{~h}$ of incubation, which was used quickly after $10 \mathrm{~h}$ of incubation, and completely depleted by $24 \mathrm{~h}$ (Table S1).

As compared to GOS, FOS and 2'-FL, inulin (DP2-60, distribution of DPs in Fig. S11) and IMMP are composed of longer chains, the degradation of which is visualized by HPAEC and HPSEC elution chromatograms (Fig. 3). Specifically, the inulin degradation kinetics differed between subjects (Fig. 3A). For example, the degradation of inulin was fastest by the microbiota of subject AD03 and slowest by that of AD06. In addition, inulin degradation was nearly completed after $24 \mathrm{~h}$ of incubation, whereas degradation of FOS only took approximately $10 \mathrm{~h}$ (Figs. 2C and 3A). Moreover, shorter DPs from inulin were more quickly degraded than the longer ones by the microbiota of some subjects.

The molecular weight of IMMP used in the current study ranged from 4.84 to $36.60 \mathrm{kDa}$ (Fig. 3B). IMMP was not degraded after $4 \mathrm{~h}$ of incubation by the microbiota of any of the subjects. After $10 \mathrm{~h}, \mathrm{a}$ clear shift in molecular weight of IMMP was observed, being most pronounced in AD03, AD04 and EL06. The degradation of IMMP coincided with the formation of oligosaccharides after $10 \mathrm{~h}$ of incubation for all subjects (Fig. S12). The concentration of these oligosaccharides was considerably higher in case of EL05 and EL06, compared to the incubations with faeces obtained from other subjects. After $24 \mathrm{~h}$, the big shoulder containing large IMMP 
A

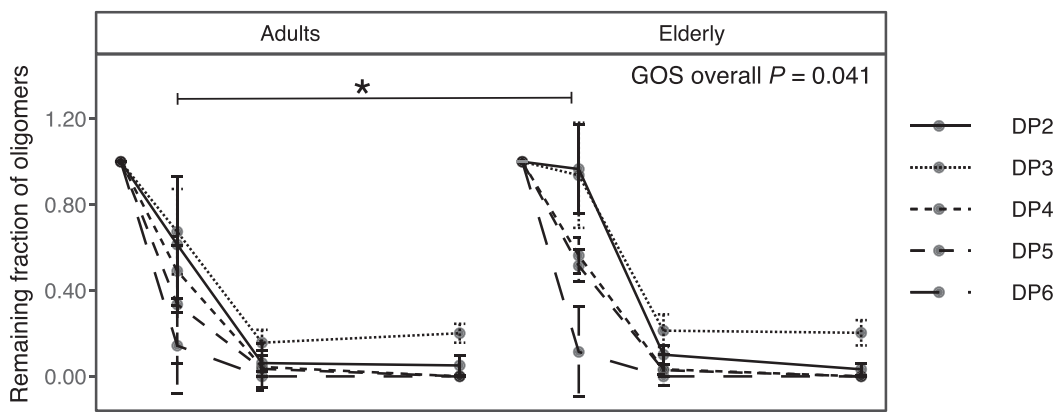

B
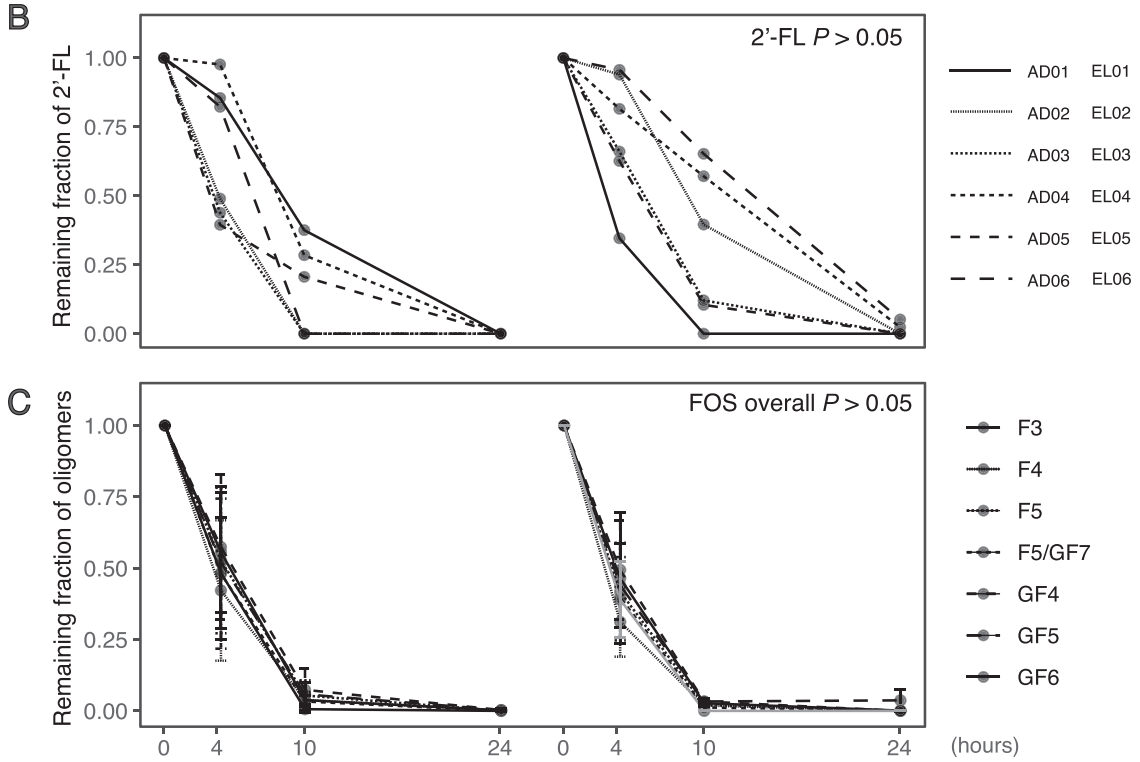

Fig. 2 Degradation kinetics of different carbohydrates. A GOS, B 2'-FL and C FOS. Data are expressed as fraction of residual substrate as compared to the initial concentration of oligosaccharides or $2^{\prime}$-FL. Concentrations per DP in initial GOS and FOS were set to 1.0. Mean \pm SD are shown (A and $\mathbf{C})$. Error bar was included to demonstrate the variability between subjects. In (B) no error bar was added as individual data (per subject) was shown. Individual data for $\mathbf{A}$ and $\mathbf{C}$ are available in Figs. S9 and S10. AD adult, EL elderly, DP degree of polymerization, GOS galacto-oligosaccharides, FOS fructo-oligosaccharides, 2'-FL 2'-fucosyllactose, F fructose, G glucose, SD standard deviation.

molecules (6.10-36.60 kDa, Fig. 3B) and newly formed IMMP derived oligosaccharides had nearly disappeared for all subjects (Fig. S12).

\section{Effect of transport and storage on the viability of the microbiota in faecal samples}

PERMANOVA based on weighted and unweighted UniFrac distance matrices did not reveal significant differences between the microbiota of directly frozen faeces and that of cryo-conserved faecal inocula with or without PMA treatment (Fig. S13A, B). Although the relative abundance of some bacterial groups (Table S2), such as Bacteroidaceae and Prevotellaceae (Fig. S13C), differed in some subjects, visually reflected in larger weighted as compared to unweighted distances, comparative analysis did not show significant differences in these microbial groups. Quantification of total 16S rRNA gene copy numbers demonstrated lower fraction of microbes in the PMA treated samples (Table S3), which is in line with a previous study reporting that up to one-third of microbes in the faeces consist of dead cells [32]. Although acknowledging the limitation of the PMA analyses [33], overall, our results demonstrated a good recovery of microbes with the anoxic cryo-conservation protocol used to transport and store the samples for incubation studies.

\section{Changes in microbiota composition in presence of different carbohydrates \\ 16S rRNA gene sequencing and subsequent analyses were done} on duplicate samples, which show high sequencing reproducibility with Pearson similarity index 0.9880 $[0.9740,0.9953]$ (median and interquartile range) (Table S4). PERMANOVA using weighted UniFrac distances revealed significant contribution of age group (4.87\%), subject identity $(32.35 \%)$, type of carbohydrate $(10.43 \%)$ and sampling time point $(18.45 \%)$ to the overall microbiota variation (Fig. 1B), while the contribution of these factors was $8.06 \%, 64.50 \%, 1.86 \%$ and $8.44 \%$, respectively, based on unweighted UniFrac distances (Fig. 1C).

Variation partitioning per sampling time point demonstrated significant contribution of age group $(P<0.01)$ throughout the incubation period, although its contribution remained low (5.87-9.86\%) compared to that of subject identity and carbohydrate (Fig. 1E, F). Based on weighted and unweighted UniFrac distance matrices, variation explained by subject identity was highest at $\mathrm{Oh}(85.76 \%$ and $87.47 \%$, respectively) and decreased gradually towards $35.78 \%$ or $69.56 \%$, respectively, over time. In contrast, the type of carbohydrate did not contribute to the microbiota variation at the start of the incubation as expected, whereas with the progress of incubation, its contribution increased to $38.07 \%$ (weighted UniFrac) or $7.26 \%$ (unweighted UniFrac) at the end of the incubation. Moreover, during the incubation, the microbial diversity and richness decreased significantly both in the microbiota of adults and that of elderly (Fig. S14 and Table S5) indicative for a selective stimulation exerted by the different carbohydrates during the incubation.

During the incubation, in comparison to non-carbohydrate controls, a large number of genera changed in their relative 


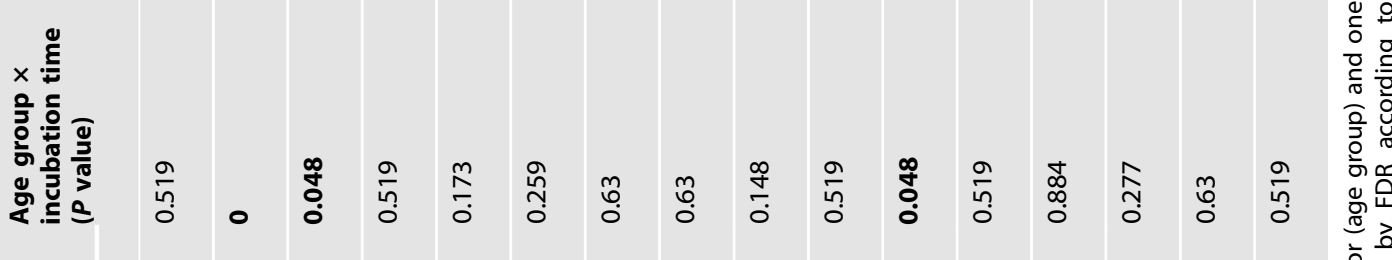

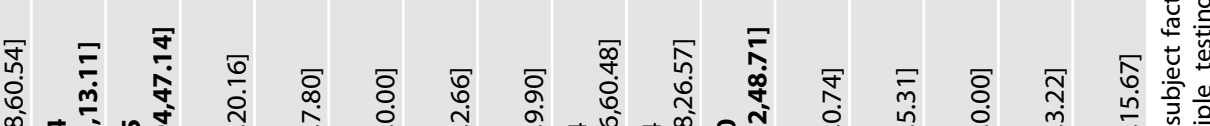

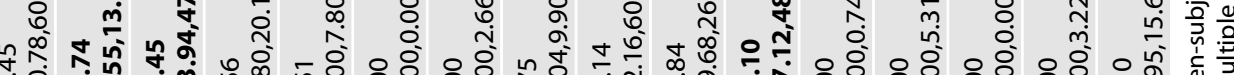

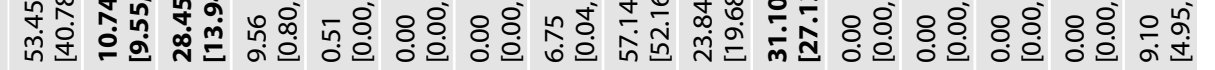

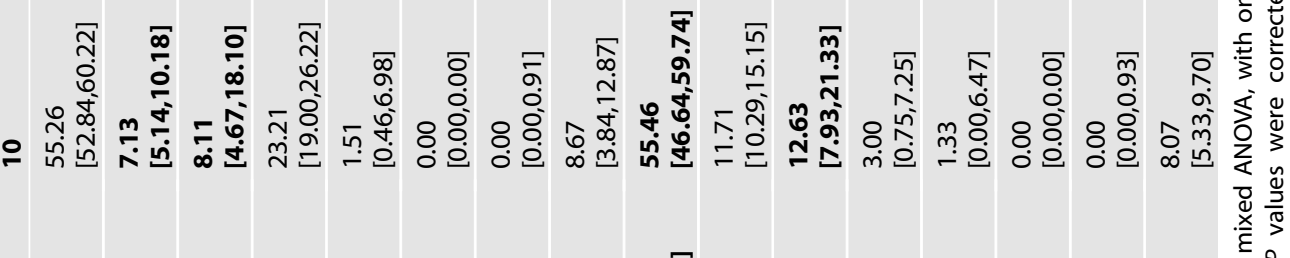

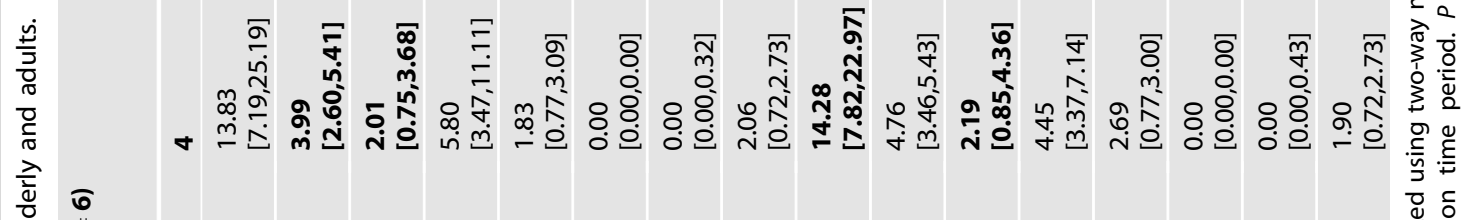

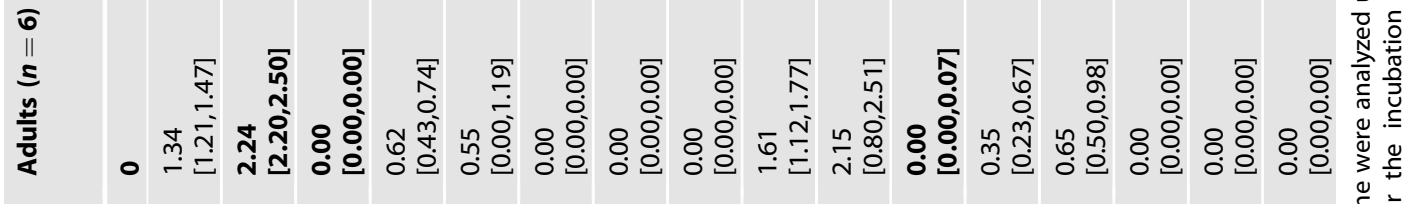

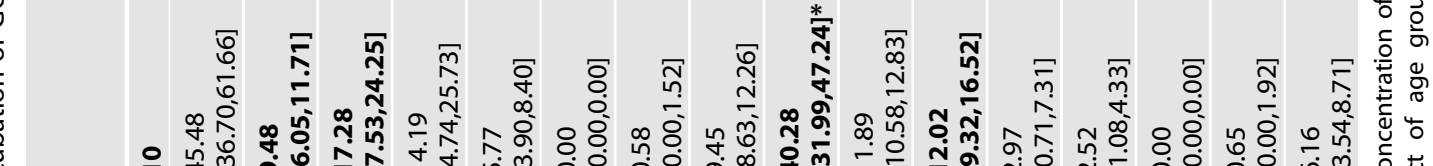

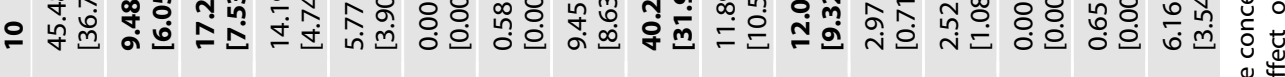

(1)




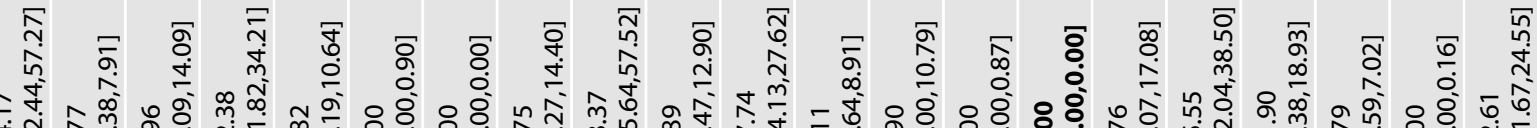

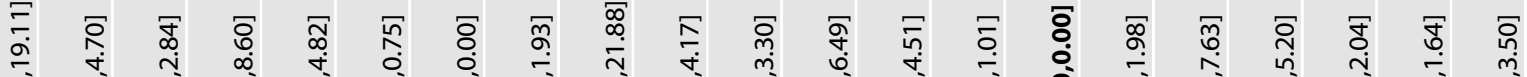

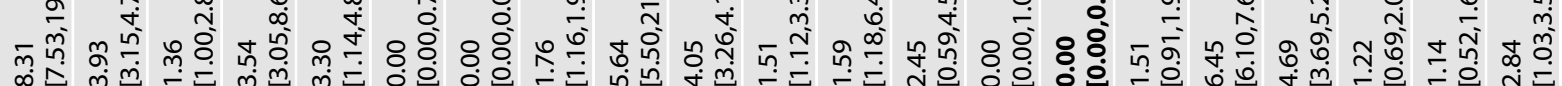
m mu-U mu me ó 0

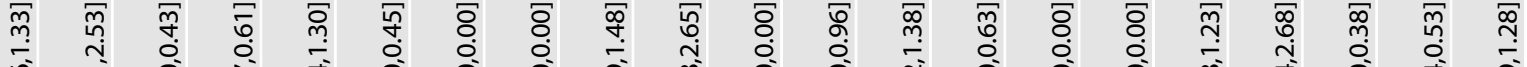
и ๘

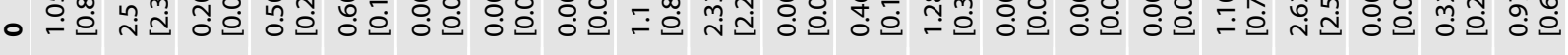

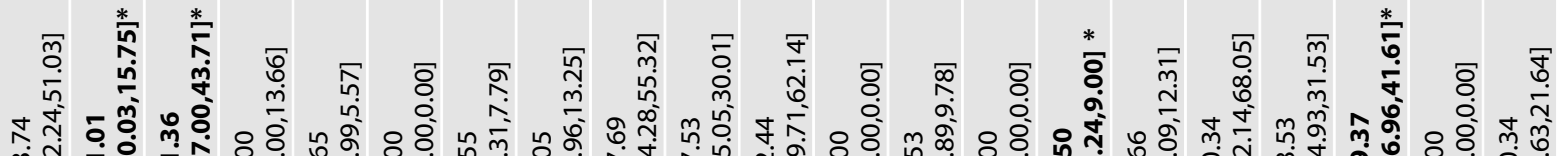

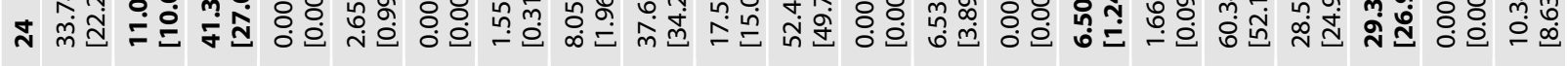

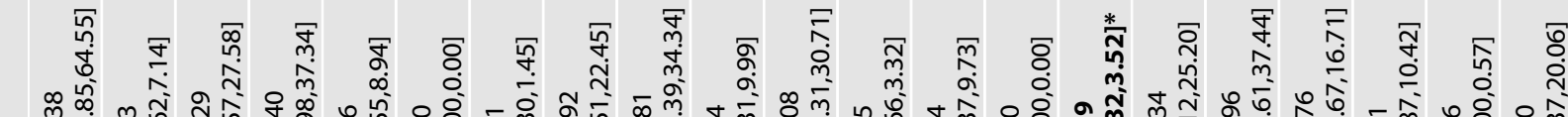

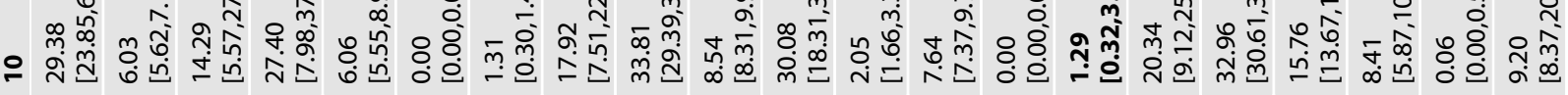

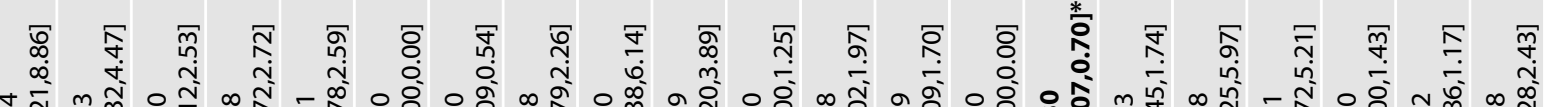
m心

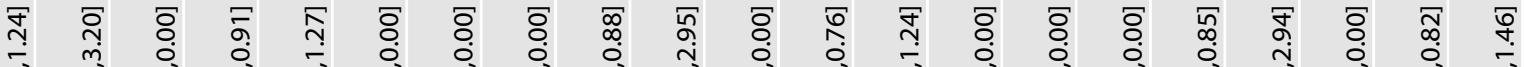
К

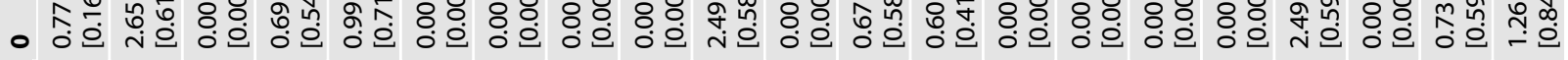

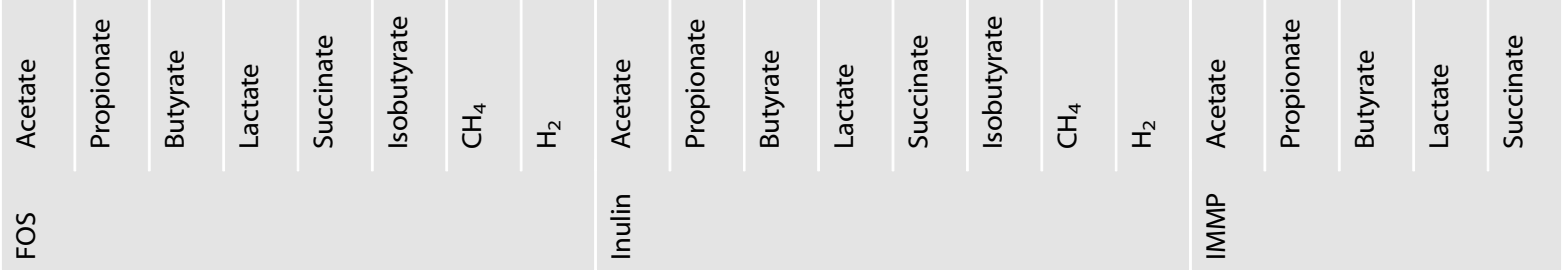


abundance in response to different carbohydrates, while altered genera differed between carbohydrates (Figs. S4 and S5). Among other genera, Bifidobacterium relative abundance increased the most in response to FOS, 2'-FL, GOS and inulin. Notably, compared to other carbohydrates, the increase in the relative abundance of Bifidobacterium was largest in response to GOS (Figs. S4F and S5F). This alteration was in general more pronounced in the microbiota of adults than that of elderly, with few exceptions (Fig. S6A). Interestingly, in response to inulin, the microbiota of adults and elderly changed differently, i.e., inulin supplementation lifted the relative abundance of Bifidobacterium in the adults (Fig. S4D), while it promoted the relative abundance of Blautia in the pre-frail elderly (Fig. S5D). Moreover, in the presence of IMMP, the relative abundance of Bacteroides increased over time both in adults and elderly (Figs. S4E, S5E and S6B). Finally, the relative abundance of Dorea and Coprococcus 3 mainly significantly increased in the noncarbohydrate controls, except for the microbiota of EL06, which also showed an increased level of Dorea in the presence of all five studied carbohydrates (Figs. S4, S5, and S6C).

To assess how well the incubation of faecal microbiota in the presence of carbohydrates in vitro reflects the in vivo observations, we compared microbiota composition and its dynamics over time between the in vitro GOS incubations and the in vivo GOS effects [11] of the same subjects (Figs. 4, S15, S16 and Table S6). Although we realize that the timelines between in vivo and in vitro are completely different, this comparison suggests that incubation of carbohydrates in vitro can to some level mimic the in vivo observations with respect to alterations in microbiota composition (Figs. 4A and S17), especially its impact on the relative abundance of Bifidobacterium (Fig. 4A). In two subjects, i.e. EL02 and EL06 (Figs. $4 \mathrm{~B}$ and $\mathrm{S} 15$ ), the relative abundance of Bifidobacterium was lower or even below the detection threshold at the start of the incubation, with incongruent alterations in Bifidobacterium levels in response to GOS (Figs. 4B, S6A and S15). The relative abundance of Bifidobacterium in EL02 increased after one week of GOS supplementation, whereas in vitro only a subtle increase was observed (Figs. S15 and S16). In contrast, for EL06, the microbiota did not have detectable levels of Bifidobacterium at baseline both in vitro and in vivo. After 1 week of GOS intervention, the relative abundance of Bifidobacterium increased while, in contrast to other elderly, it completely disappeared after 4 weeks GOS supplementation (Fig. S15). Remarkably, in line with this observation, the relative abundance of Bifidobacterium stayed under the detection limit during the in vitro incubations with faecal inoculum from this subject (Figs. 4B and S16). Overall, after 4 weeks of GOS intervention in vivo, the microbiota was more similar to the in vitro profiles after $10 \mathrm{~h}$ of GOS incubation (Figs. 4A and S17), compared to that after $10 \mathrm{~h}$ of $2^{\prime}-\mathrm{FL}$ or protein incubation.

\section{DISCUSSION}

In the current study, we compared the metabolic capacity of faecal microbiota obtained from adults and pre-frail elderly during an in vitro incubation study in medium mimicking the intestinal lumen and using carbohydrates varying in molecular structure as carbon and energy source. We hypothesized that the microbiota of pre-frail elderly is less efficient in carbohydrate degradation and metabolite production. We found that a significant fraction of the microbiota variation and metabolite production could be explained by age group, although the different subjects and carbohydrates explained most of the variation. Our data on microbiota composition and metabolite production supported the notion that the metabolic capacity of carbohydrate degradation by the microbiota of pre-frail elderly differed from that of healthy adults, with some carbohydrates being degraded at a decreased rate in the former group.

Although the overall dietary or fibre intake and the health status of the pre-frail elderly in this study were similar to that of adults 

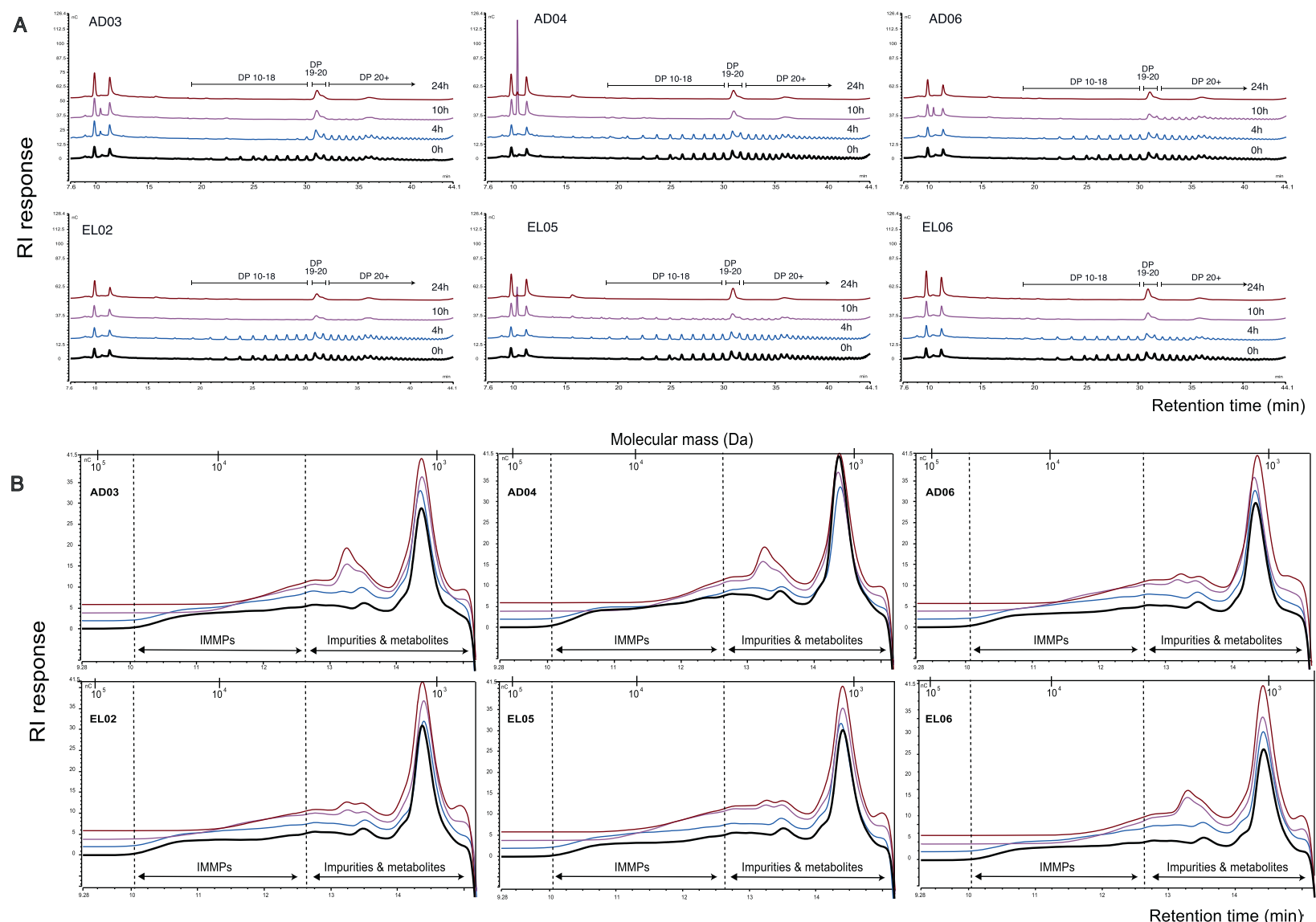

Fig. 3 Degradation kinetics of Inulin and IMMP. A HPAEC elution patterns of Inulin. B HPSEC patterns of IMMP before and after incubation with faecal microbiota of three adults and three elderly. AD adult, EL elderly, DP degree of polymerization, IMMP isomalto/maltopolysaccharides. Incubation lasted for $24 \mathrm{~h}$. Samples were taken at $0 \mathrm{~h}$ (Black line), $4 \mathrm{~h}$ (Blue line), $10 \mathrm{~h}$ (Purple line) and $24 \mathrm{~h}$ (Brown line). As duplicate samples demonstrated very high reproducibility, hereby only the chromatography elution pattern of one sample (out of the duplicate) is shown.

based on comorbidity, immune and oxidative stress markers [11], the lower relative abundance of Bifidobacterium in elderly was evident. This is in line with earlier observations that the microbiota of elderly is characterized by lower relative abundance of Bifidobacterium compared to that of healthy adults as recently reviewed [10]. The genomes of Bifidobacterium spp. contain a large number of genes encoding carbohydrate modification enzymes, such as glycosyl hydrolases, $A B C$ transporters and the phosphoenolpyruvate-phosphotransferase system, and hence, bifidobacteria act actively in carbohydrate degradation and utilization [34]. In the current study, decreased relative abundance of Bifidobacterium in the microbiota of pre-frail elderly at the start of the incubation, as well as a less uniform/consistent increase in Bifidobacterium relative abundance during incubation compared to that in healthy adults, could have contributed to the declined efficiency in the carbohydrate degradation, especially for GOS [35], 2'-FL [36] and inulin [37], which are known as bifidogenic ingredients. Collectively, it is possible that a lower efficiency in degradation of some bifidogenic components could be a sign of changed intestinal conditions in pre-frail elderly. Nevertheless, it is hard to decipher cause-consequence relations and that our statement remains speculative. Moreover, the impact of lower Bifidobacterium (relative) abundance on the intestinal physiology during the ageing process remains to be further explored.

We observed differences in altered genera during the incubation of different carbohydrates. Although in line with other studied carbohydrates with respect to the observed increase in the level of Bifidobacterium during the incubation, the presence of IMMP was particularly associated with an increase of Bacteroides, which was accompanied with increased concentration of succinate in both age groups. Consistently, in addition to the increase in the relative abundance of Bifidobacterium, $\mathrm{Gu}$ et al. observed an increase in the relative abundance of Bacteroides and the concentration of succinate during in vitro incubation of IMMP with adult faecal microbiota [20]. Generally, only low concentrations of succinate are observed in the human intestine [38] as an intermediate in the synthesis of propionate, a common product of Bacteroides or Veillonellaceae through the succinate pathway [39]. In the current study, a significant increase in the relative abundance of Bacteroides resulted in the accumulation of succinate. As reviewed by Fernandez-Veledo et al. [40], several recent studies in humans and in mice have suggested to treat obesity and related co-morbidities via modulating the succinate level in the intestine. Hence, the IMMP studied here could be a potential candidate for intervention studies to investigate its direct and indirect impact on human health. On the other hand, although no significant difference in succinate accumulation between adults and elderly were observed, it seems that the higher succinate production in AD03, AD04 and EL06 coincided with faster acidification when compared to the other subjects. Similar observations were made for lactate production and faster acidification in the GOS and FOS incubations. These results could imply that differences in initial $\mathrm{pH}$ drop could play a role in subsequent microbial activity including the conversion of 
A

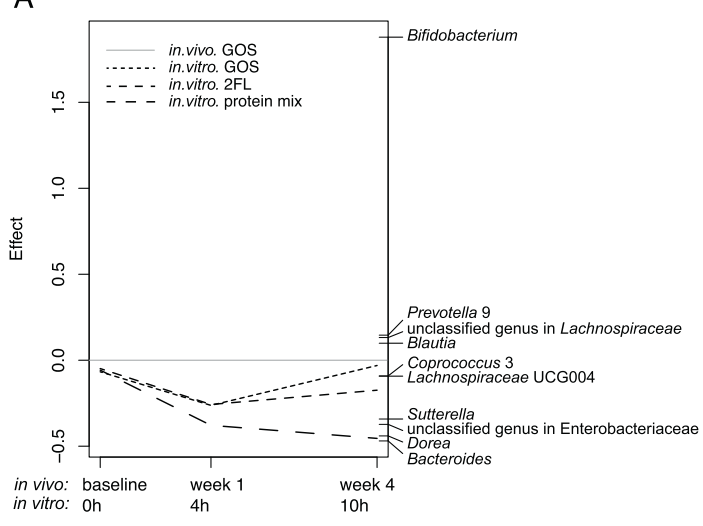

B

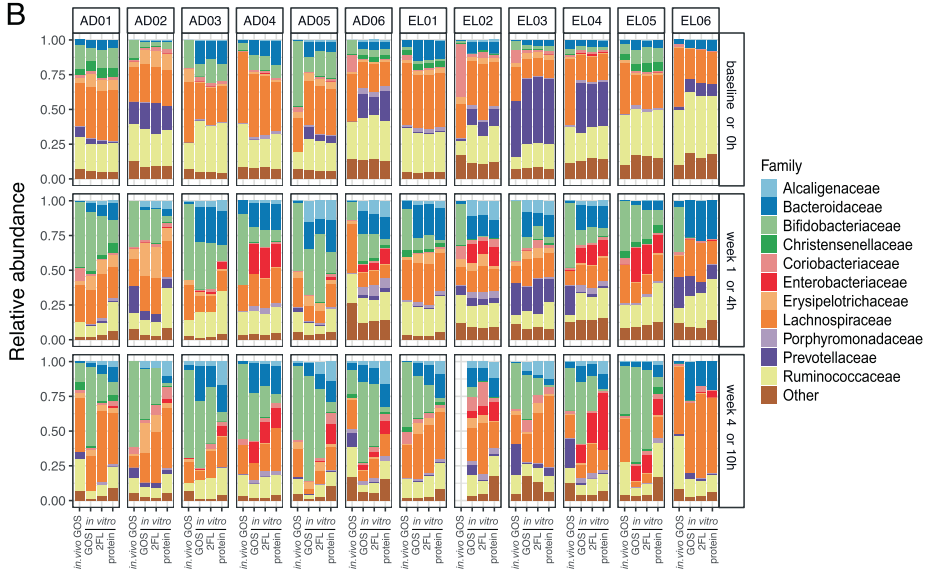

Fig. 4 Comparative analysis between in vitro and in vivo [11] including both adults and elderly. A First axis of the principal response curve showing alterations in microbial composition over time in response to GOS in vivo and in response to non-carbohydrate control (protein mix) and carbohydrates (GOS or 2'-FL) in vitro, while taking in vivo GOS intervention as reference. Both duplicate samples were included for the analysis. Genera for which the model best explained the observed variation between reference and treatments (weights $>0.05$ ) are shown on the right side of the figure. B Relative abundance of different bacterial families (top 12, ranked based on the average relative abundance across the entire dataset) in the microbiota of six adults and six elderly. Averaged relative abundance of the duplicate samples was used here (B) for visibility. Top 12 microbial families are listed in the legend. Other families are summarized as "Other". Each column represents the corresponding type of sample from one subject. Samples collected at $24 \mathrm{~h}$ were excluded from this comparative analysis as some carbohydrates were completely depleted within $10 \mathrm{~h}$ (see Figs. 2 and 3). AD adult, EL elderly, GOS galacto-oligosaccharides, 2'-FL 2'fucosyllactose.

lactate to butyrate, which has been observed previously in in vitro study setups $[41,42]$. Consistent with the observations in carbohydrate degradation, the dynamics of metabolite production over time differed significantly between adults and pre-frail elderly, in addition to the effect of carbohydrate- and subjectspecificity. On one hand, this is in line with the high individuality shown in the microbiota composition. For instance, only incubations with faecal inocula obtained from subjects with detectable levels of methanogens i.e., Methanobrevibacter, demonstrated $\mathrm{CH}_{4}$ production, further reinforcing individual differences in metabolic capacity of the microbiota. On the other hand, our data also emphasized the differences in microbial composition between age groups. Compared to healthy adults, the microbiota of pre-frail elderly had lower levels of Bifidobacterium, and as such was slower in $2^{\prime}$-FL utilization and acetate production at the beginning of the incubation [43]. At later time points, compared to healthy adults, incubation with microbiota of pre-frail elderly had higher concentrations of propionate and butyrate, indicating the presence of propionate and butyrate-producing microbes like Coprococcus [43]. The relative abundance of the latter genus was also higher in pre-fail elderly than in adults at the start of the incubation. Some of the other carbohydrates, such as FOS and inulin, did not show significant differences in their degradation rate between the age groups. This could be attributed to the existence of FOS and/or inulin in daily diet (e.g., wheat, banana and onion). Therefore, the elderly microbiota may not need to adapt to their degradation, as such could use them to produce the main SCFAs. Interestingly, in response to inulin Blautia was increased in abundance in elderly versus Bifidobacterium in young adults. Bifidobacterial effects have been observed in elderly human intervention [44]. Perhaps in this in vitro gut model, the low levels of bifidobacteria in elderly faeces allowed metabolism of the long chains of inulin by other microbes, which has been reported [45], and in this case Blautia. Furthermore, the differences in metabolites between age groups varied for different carbohydrates, which could in part be attributed to the differences in carbohydrates' physical and chemical properties [19], although it remains challenging to explain comprehensibly how carbohydrate properties affect microbiota composition, metabolite production, as well as the corresponding direct/indirect impact on health.

We clearly showed that all carbohydrates were utilized over time. In some cases (e.g., 2'-FL and IMMP) we also observed that some of the remaining cryoprotective agent glycerol was utilized over time. Previous studies have shown that glycerol could possibly favour some bacterial growth and corresponding metabolism [46]. Although we never saw a preference of the community for glycerol over the carbohydrate, we realize that it is a potential additional carbon source in our system, and therefore, we cannot exclude its impact on the microbiota composition and metabolite profiles in the cases in which we observed its utilization. This general drawback of cultivation experiments with microbial communities is difficult to circumvent as cryoprotection is crucial when samples are collected for in vitro cultivation experiments, in addition to the limitation of medium dependency and selectivity.

We performed the in vitro incubation of different carbohydrates using faecal inocula of a subgroup of subjects involved in a GOS intervention study. Comparative analyses revealed that the GOS intervention data best matched with data from the GOS incubation in the in vitro experiment, conferring that in vitro approaches can at some level offer a good model for in vivo observations. Moreover, we demonstrated a large contribution of inter-individual differences in microbiota composition, and microbial capacity with respect to carbohydrate degradation and metabolite production, which collectively underscored the importance of taking individual-specific differences into account in future studies.

In conclusion, the efficacy of carbohydrate degradation by the microbiota of pre-frail elderly differed from that of healthy adults, with some carbohydrates showing significantly decreased efficacy of degradation by the microbiota of pre-frail elderly. Although the pre-frail elderly showed no physical and immune decline yet [11], the lower level of Bifidobacterium and their lower involvement in carbohydrate degradation made us speculate that a microbial change with declined efficacy of certain prebiotic carbohydrates degradation may be expected in the microbiota of pre-frail elderly. Whether this has an impact on the progress of frailty or other health parameters in elderly and whether specific (dietary) 
interventions can postpone this process is speculative and could be addressed in follow-up studies.

\section{REFERENCES}

1. Nicholson JK, Holmes E, Kinross J, Burcelin R, Gibson G, Jia W, et al. Host-gut microbiota metabolic interactions. Science. 2012;336:1262-7.

2. O'Toole PW, Jeffery IB. Gut microbiota and aging. Science. 2015;350:1214-5.

3. Mueller S, Saunier K, Hanisch C, Norin E, Alm L, Midtvedt T, et al. Differences in fecal microbiota in different European study populations in relation to age, gender, and country: a cross-sectional study. Appl Environ Microbiol. 2006;72:1027-33.

4. Biagi E, Nylund L, Candela M, Ostan R, Bucci L, Pini E, et al. Through ageing, and beyond: gut microbiota and inflammatory status in seniors and centenarians. PLOS ONE. 2010;5:e10667.

5. Woodmansey EJ, McMurdo ME, Macfarlane GT, Macfarlane S. Comparison of compositions and metabolic activities of fecal microbiotas in young adults and in antibiotic-treated and non-antibiotic-treated elderly subjects. Appl Environ Microbiol. 2004;70:6113-22.

6. Tiihonen K, Tynkkynen S, Ouwehand A, Ahlroos T, Rautonen N. The effect of ageing with and without non-steroidal anti-inflammatory drugs on gastrointestinal microbiology and immunology. Br J Nutr. 2008;100:130-7.

7. Biagi E, Franceschi C, Rampelli S, Severgnini M, Ostan R, Turroni S, et al. Gut microbiota and extreme longevity. Curr Biol. 2016;26:1480-5.

8. Clooney AG, Fouhy F, Sleator RD, O' Driscoll A, Stanton C, Cotter PD, et al. Comparing apples and oranges?: next generation sequencing and its impact on microbiome analysis. PLoS ONE. 2016;11:e0148028.

9. Santoro A, Ostan R, Candela M, Biagi E, Brigidi P, Capri M, et al. Gut microbiota changes in the extreme decades of human life: a focus on centenarians. Cell Mol Life Sci. 2018;75:129-48.

10. An R, Wilms E, Masclee A, Smidt H, Zoetendal EG, Jonkers D. Age-dependent changes in $\mathrm{Gl}$ physiology and microbiota: time to reconsider? Gut. 2018;67:2213-22.

11. Wilms E, An R, Smolinska A, Stevens $Y$, Weseler AR, Elizalde M, et al. Galactooligosaccharides supplementation in prefrail older and healthy adults increased faecal bifidobacteria, but did not impact immune function and oxidative stress. Clin Nutr. 2021;40:3019-31.

12. Rampelli $S$, Candela $M$, Turroni $S$, Biagi $E$, Collino $S$, Franceschi $C$, et al. Functional metagenomic profiling of intestinal microbiome in extreme ageing. Aging. 2013;5:902-12.

13. Louca S, Polz MF, Mazel F, Albright M, Huber JA, O'Connor MI, et al. Function and functional redundancy in microbial systems. Nat Ecol Evol. 2018;2:936-43.

14. Flint HJ, Scott KP, Duncan SH, Louis P, Forano E. Microbial degradation of complex carbohydrates in the gut. Gut Microbes. 2012;3:289-306.

15. Mitchell A, Romano GH, Groisman B, Yona A, Dekel E, Kupiec M, et al. Adaptive prediction of environmental changes by microorganisms. Nature. 2009:460:220-4.

16. Macfarlane GT, Steed $H$, Macfarlane S. Bacterial metabolism and health-related effects of galacto-oligosaccharides and other prebiotics. J Appl Microbiol. 2008;104:305-44.

17. Thurl S, Munzert M, Boehm G, Matthews C, Stahl B. Systematic review of the concentrations of oligosaccharides in human milk. Nutr Rev. 2017;75:920-33.

18. Salli K, Anglenius H, Hirvonen J, Hibberd AA, Ahonen I, Saarinen MT, et al. The effect of 2'-fucosyllactose on simulated infant gut microbiome and metabolites; a pilot study in comparison to GOS and lactose. Sci Rep. 2019;9:13232.

19. Wang $M$, Wichienchot $S$, He $X$, Fu X, Huang $Q$, Zhang B. In vitro colonic fermentation of dietary fibers: Fermentation rate, short-chain fatty acid production and changes in microbiota. Trends Food Sci Technol. 2019;88:1-9.

20. Gu F, Borewicz K, Richter B, der Zaal PH, Smidt H, Buwalda PL, et al. In vitro fermentation behavior of isomalto/malto-polysaccharides using human fecal inoculum indicates prebiotic potential. Mol Nutr Food Res. 2018;62: e1800232.

21. Leemhuis H, Dobruchowska JM, Ebbelaar M, Faber F, Buwalda PL, van der Maarel MJEC, et al. Isomalto/malto-polysaccharide, a novel soluble dietary fiber made via enzymatic conversion of starch. J Agric Food Chem. 2014;62:12034-44.

22. Parada AE, Needham DM, Fuhrman JA. Every base matters: assessing small subunit rRNA primers for marine microbiomes with mock communities, time series and global field samples. Environ Microbiol. 2016;18:1403-14.

23. Apprill A, McNally S, Parsons R, Weber L. Minor revision to V4 region SSU rRNA 806R gene primer greatly increases detection of SAR11 bacterioplankton. Aquat Microb Ecol. 2015;75:129-37.

24. An R, Wilms E, Smolinska A, Hermes GDA, Masclee AAM, de Vos P, et al. Sugar beet pectin supplementation did not alter profiles of fecal microbiota and exhaled breath in healthy young adults and healthy elderly. Nutrients. 2019;11:2193.
25. Ramiro-Garcia J, Hermes GDA, Giatsis C, Sipkema D, Zoetendal EG, Schaap PJ, et al. NG-Tax, a highly accurate and validated pipeline for analysis of $16 \mathrm{~S}$ rRNA amplicons from complex biomes. F1000Research. 2016;5:1791.

26. Quast C, Pruesse E, Yilmaz P, Gerken J, Schweer T, Yarza P, et al. The SILVA ribosomal RNA gene database project: improved data processing and web-based tools. Nucleic Acids Res. 2013;41:D590-596.

27. Yilmaz P, Parfrey LW, Yarza P, Gerken J, Pruesse E, Quast C, et al. The SILVA and "All-species Living Tree Project (LTP)" taxonomic frameworks. Nucleic Acids Res. 2014;42:D643-648.

28. Nocker A, Cheung CY, Camper AK. Comparison of propidium monoazide with ethidium monoazide for differentiation of live vs. dead bacteria by selective removal of DNA from dead cells. J Microbiol Methods. 2006;67:310-20.

29. McMurdie PJ, Holmes S. phyloseq: an R package for reproducible interactive analysis and graphics of microbiome census data. PLoS ONE. 2013;8:e61217.

30. Oksanen J, Blanchet FG, Kindt R, Legendre P, Minchin PR, O'Hara RB, et al. Package 'vegan'. Community ecology package, version. 2013;2. CRAN.

31. Kassambara A. rstatix: pipe-friendly framework for basic statistical test. R package version 0.5.0. CRAN

32. Ben-Amor K, Heilig H, Smidt H, Vaughan EE, Abee T, de Vos WM. Genetic diversity of viable, injured, and dead fecal bacteria assessed by fluorescence-activated cell sorting and 16S rRNA gene analysis. Appl Environ Microbiol. 2005;71:4679-89.

33. Wang Y, Yan Y, Thompson KN, Bae S, Accorsi EK, Zhang Y, et al. Whole microbial community viability is not quantitatively reflected by propidium monoazide sequencing approach. Microbiome. 2021;9:1-13.

34. Pokusaeva K, Fitzgerald GF, van Sinderen D. Carbohydrate metabolism in Bifidobacteria. Genes Nutr. 2011;6:285-306.

35. Slavin J. Fiber and prebiotics: mechanisms and health benefits. Nutrients. 2013;5:1417-35.

36. Bode L. Human milk oligosaccharides: prebiotics and beyond. Nutr Rev. 2009;67: S183-191.

37. Meyer D, Stasse-Wolthuis M. The bifidogenic effect of inulin and oligofructose and its consequences for gut health. Eur J Clin Nutr. 2009;63:1277-89.

38. Cummings J, Pomare E, Branch W, Naylor C, Macfarlane G. Short chain fatty acids in human large intestine, portal, hepatic and venous blood. Gut. 1987;28:1221-7.

39. Reichardt N, Duncan SH, Young P, Belenguer A, McWilliam Leitch C, Scott KP et al. Phylogenetic distribution of three pathways for propionate production within the human gut microbiota. The ISME J. 2014;8:1323-35.

40. Fernandez-Veledo S, Vendrell J. Gut microbiota-derived succinate: Friend or foe in human metabolic diseases? Rev Endocr Metab Disord. 2019;20:439-47.

41. Ilhan ZE, Marcus AK, Kang D-W, Rittmann BE, Krajmalnik-Brown R. pH-mediated microbial and metabolic interactions in fecal enrichment cultures. Msphere. 2017;2:e00047-17.

42. Duncan $\mathrm{SH}$, Louis $\mathrm{P}$, Thomson JM, Flint $\mathrm{HJ}$. The role of $\mathrm{pH}$ in determining the species composition of the human colonic microbiota. Environ Microbiol. 2009;11:2112-22.

43. Koh A, De Vadder F, Kovatcheva-Datchary P, Bäckhed F. From dietary fiber to host physiology: short-chain fatty acids as key bacterial metabolites. Cell. 2016;165:1332-45.

44. Kiewiet MBG, Elderman ME, El Aidy S, Burgerhof J, Visser $\mathrm{H}$, Vaughan EE, et al. Flexibility of gut microbiota in ageing individuals during dietary fiber long-chain inulin intake. Mol Nutr Food Res. 2021;65:e2000390.

45. Scott KP, Martin JC, Duncan SH, Flint HJ. Prebiotic stimulation of human colonic butyrate-producing bacteria and bifidobacteria, in vitro. FEMS Microbiol Ecol. 2014;87:30-40.

46. De Weirdt R, Possemiers S, Vermeulen G, Moerdijk-Poortvliet TC, Boschker HT, Verstraete W, et al. Human faecal microbiota display variable patterns of glycerol metabolism. FEMS Microbiol Ecol. 2010;74:601-11.

\section{ACKNOWLEDGEMENTS}

This study was funded by the public-private partnership 'CarboKinetics' coordinated by the Carbohydrate Competence Centre (CCC), as part of the NWO-CCC partnership programme. Participating industrial partners are Agrifirm Innovation Center BV, FrieslandCampina Nederland BV, Cooperatie AVEBE UA, DSM Food Specialties BV, Nutrition Sciences NV, Sensus BV and VanDrie Holding NV. NWO: Netherlands Organisation for Scientific Research.

\section{AUTHOR CONTRIBUTIONS}

The authors contributions were as follows: Conceptualization, EGZ, HS, DMAEJ, AAMM and RA; Funding acquisition, EGZ, HS, DMAEJ, and AAMM; Resources, AAMM, HS and HAS; Investigation, RA and EW; Methodology, MJL and MPHT; Writing original draft, RA. 


\section{COMPETING INTERESTS}

The authors declare no competing interests.

\section{ADDITIONAL INFORMATION}

Supplementary information The online version contains supplementary material available at https://doi.org/10.1038/s43705-021-00065-5.

Correspondence and requests for materials should be addressed to Ran An.

Reprints and permission information is available at http://www.nature.com/reprints

Publisher's note Springer Nature remains neutral with regard to jurisdictional claims in published maps and institutional affiliations. (i) Open Access This article is licensed under a Creative Commons Attribution 4.0 International License, which permits use, sharing, adaptation, distribution and reproduction in any medium or format, as long as you give appropriate credit to the original author(s) and the source, provide a link to the Creative Commons license, and indicate if changes were made. The images or other third party material in this article are included in the article's Creative Commons license, unless indicated otherwise in a credit line to the material. If material is not included in the article's Creative Commons license and your intended use is not permitted by statutory regulation or exceeds the permitted use, you will need to obtain permission directly from the copyright holder. To view a copy of this license, visit http://creativecommons. org/licenses/by/4.0/.

(c) The Author(s) 2021 